\title{
Combined hamartoma of the retina and retinal pigment epithelium
}

\author{
Kanmin Xue, ${ }^{1}$ Faye Mellington, ${ }^{2}$ Irina Gout, ${ }^{1}$ Sofia Rokerya, ${ }^{1}$ Oyinkan Ibironke Olurin, ${ }^{1}$ Ahmed El-Amir ${ }^{1}$
}

${ }^{1}$ Prince Charles Eye Unit, King Edward VII Hospital, Windsor, UK

${ }^{2}$ Oxford Eye Hospital, John Radcliffe Hospital, Oxford, UK

Correspondence to Dr Kanmin Xue, kanminxue@gmail.com

\section{Summary}

We report two cases of combined hamatoma of the retina and retinal pigment epithelium (CHR-RPE), illustrated with ultrasonography, optical coherence tomography, fundus fluorescein angiography and indocyanine green angiography images. CHR-RPE could clinically mimic several other retinal conditions. Failure to distinguish it from serious malignancies such as choroidal melanoma or retinoblastoma has led to unnecessary enucleation in the past. Through these case reports and a review of literature, we show the diagnostic features of CHR-RPE, its key differential diagnoses and the management options.

\section{BACKGROUND}

Combined hamatoma of the retina and retinal pigment epithelium (CHR-RPE) is a rare and generally benign ocular hamartoma. The vast majority of previously documented cases of CHR-RPE have been published prior to the advent of optical coherence tomography (OCT). In addition, there are very few examples in the published literature of clinical, OCT, fundus fluorescein angiography (FFA) and indocyanine green (ICG) angiography images of the same lesion being shown in parallel. Therefore, our cases represent a unique opportunity to present the diagnostic features of CHR-RPE with the support of a comprehensive array of imaging modalities. CHR-RPE could mimic serious malignancies such as choroidal melanoma or retinoblastoma. Failures to recognise this in the past has led to unnecessary enucleation for presumed melanoma. It is hoped that through this article, we would increase general awareness of this important retinal condition and provide up-to-date guidance regarding its diagnosis and management.

\section{CASE PRESENTATION}

\section{Case 1}

A 13-year-old boy had been noted several years prior to have a benign condition affecting his left eye that has been blind since birth. He was otherwise fit and well with no family history of ocular conditions. Visual acuity was $6 / 4$ right and perception of light left. Examination showed a left exotropia and white reflex. Funduscopy revealed a greyish elevated lesion involving the disc and macula measuring four disc diameters (figure 1A). B-scan ultrasonography and OCT were performed with results shown (figure $1 \mathrm{~B}-\mathrm{D}$ ).

\section{Case 2}

A 24-year-old man was referred to eye clinic with recent deterioration of visual acuity in his right eye associated with floaters and flashing lights. He was previously diagnosed with a benign retinal condition at the age of 19 elsewhere. He had no other significant medical history and no family history. The best-corrected vision in the affected eye was 6/12. Examination revealed an elevated greyish-white lesion involving the inferior macula with tortuosity of surrounding vessels (figure 2A). This lesion was further evaluated using B-scan, OCT, FFA and ICG angiography (figure $2 \mathrm{~B}-\mathrm{E}$ ).

CHR-RPE was diagnosed in each case.

\section{INVESTIGATIONS}

Case 1

B-scan ultrasonography and OCT were performed with results shown in figure 1 .

\section{Case 2}

B-scan ultrasonography, OCT, FFA and ICG angiography were performed with results shown in figure 2 .

These imaging modalities demonstrate some of the characteristic features expected in CHR-RPE. The margin of the lesion appears hyperpigmented due to hypertrophy of the underlying RPE which merges seamlessly into the surrounding normal RPE without any RPE atrophy, retinal exudation, haemorrhage or detachment. OCT demonstrates that the retina within the tumour is thickened and disorganised. The retinal surface is hyporeflective, undulating and associated with epiretinal or pre-retinal membrane. Subretinal fluid has been reported to develop secondary to vitreoretinal traction but is not present in our cases. ${ }^{1}$ Blood vessels within the tumour appear tortuous due to contracture of the fibrotic membranes. This is particularly well-visualised in FFA. The FFA images from case 2 show characteristic temporal sequence of changes for CHR-RPE: early blockage of choroidal flush by the hypertrophic RPE, corkscrew tortuosity of vessels within the lesion, subtle leakage of fluorescein from a network of capillaries within the tumour (due to 


\section{BMJ Case Reports}
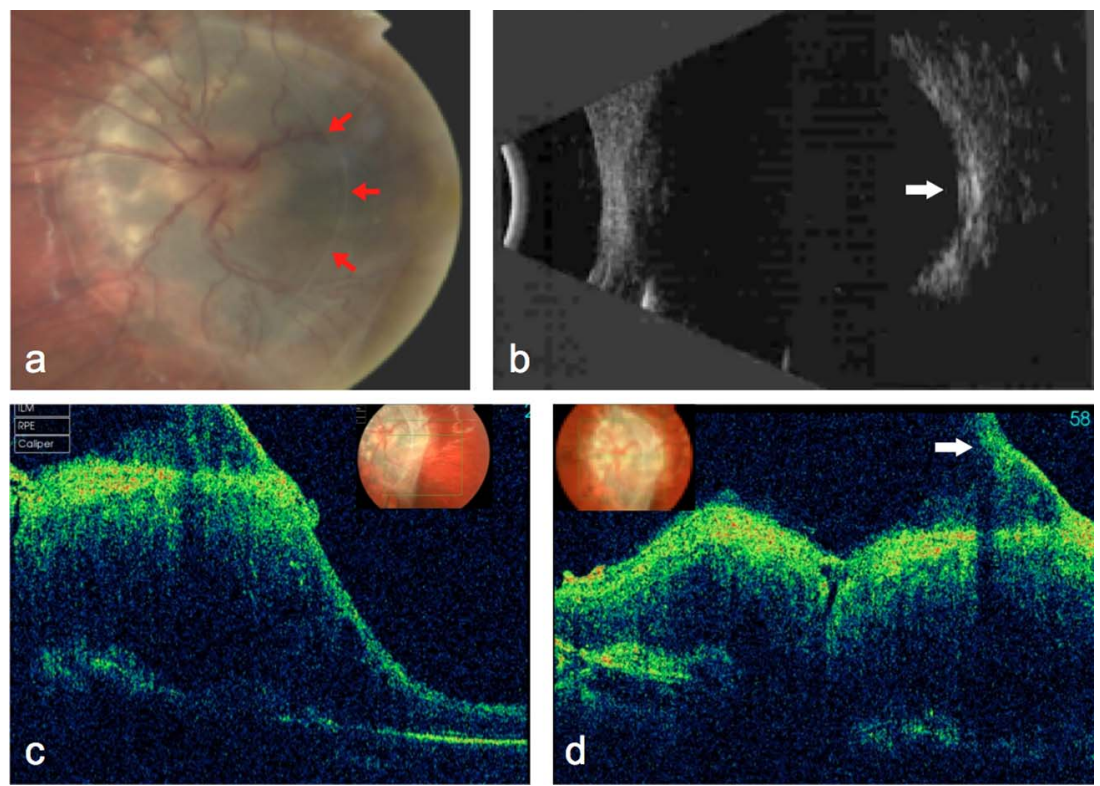

Figure 1 (A) Colour photograph of the posterior pole showed a grey elevated lesion involving the disc and macula with tortuous blood vessels. An overlying translucent fibrotic membrane was seen extending from 12-6 o'clock in a crescent configuration (red arrows).

(B) B-scan ultrasonography showed the lesion to be slightly elevated (by $1 \mathrm{~mm}$ ) with medium-to-high reflectivity (arrow). (C and D) Optical coherence tomography sections through the macula and disc, respectively, revealed thickened retina with loss of architectural layers, and a pre-retinal membrane causing vitreoretinal traction (arrow).
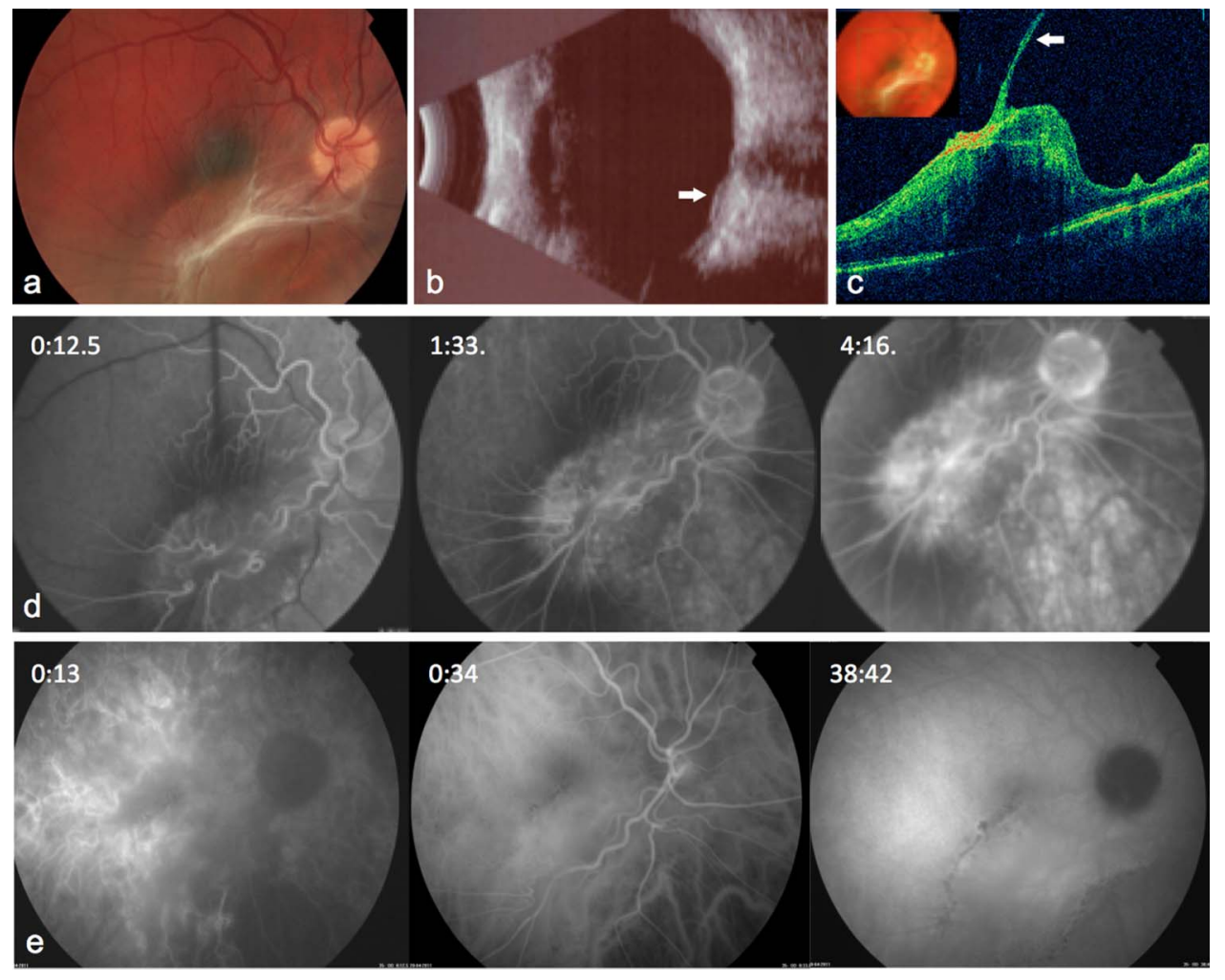

Figure 2 (A) Colour photograph of the right posterior pole showed an elevated lesion involving the inferior macula with a hyperpigmented margin which blends into the surrounding tissue. A grey-white fibrotic thickening is present over the surface of the lesion. (B) B-scan showed the lesion (arrow) to be slightly elevated with low-to-medium reflectivity. (C) Optical coherence tomography section through the centre of the lesion showed grossly thickened and disorganised retina with a tractional pre-retinal membrane (arrow). (D) Fundus fluorescein angiography demonstrated blockage of choroidal fluorescence by the outer pigmented portion of the lesion. Tortuosity of vessels within the lesion was prominent in the arterial phase. There was patchy dye leakage within the lesion and late staining. (E) Indocyanine green (ICG) did not reveal any significant choroidal vascular abnormality. Mild hyperfluorescence corresponding to the lesion and hypofluorescence outlining its border was seen in the late phase. 
stretching by fibrotic membranes), and late staining of the tumour.

Both patients had MRI of the head and ECG, which were both normal.

\section{DIFFERENTIAL DIAGNOSIS}

CHR-RPE is a rare benign tumour. It appears clinically as a mildly elevated grey-brown mass which is often juxtapapillary and almost always unilateral. ${ }^{2}$ It is imperative to distinguish this benign tumour from malignant chorioretinal lesions, in particular choroidal melanoma and retinoblastoma, as misdiagnoses have led to unnecessary enucleations. ${ }^{2}{ }^{3}$ Apart from some of the unique characteristics of CHR-RPE aforementioned, a choroidal melanoma is distinguishable on B-scan by virtue of its low internal reflectivity, uveal excavation and acoustic shadow posterior to the lesion. Angiographic findings in a choroidal melanoma can range from hypofluorescence due to blockage of choroidal fluorescence in small lesions to patchy hyperfluorescence and hypofluorescence followed by late staining in large melanomas. Retinoblastomas on the other hand, generally appear more homogenous and endophytic, and are associated with intraocular seeding and inflammation. Typical findings on B-scan would be an endophytic lesion with calcification and vitreous seeding.

CHR-RPE can appear similar to congenital hypertrophy of RPE, although the latter is usually flat, more darkly pigmented, and has a much more clearly defined border. Other differential diagnoses to consider include retinal haemangioma, melanocytoma, choroidal osteoma and astrocytic hamartoma.

\section{TREATMENT}

No active treatment was required in either case.

\section{OUTCOME AND FOLLOW-UP}

The patients are under periodic review and monitored through serial photography and OCT imaging.

\section{DISCUSSION}

We present two cases of CHR-RPE involving the optic disc and macula. This rare benign tumour was first reported by Gass in 1973. ${ }^{4}$ It generally presents during childhood (median age 7.5 year) with decreased vision (40\%) or strabismus (28\%). ${ }^{3}$ Histologically, CHR-RPE consists of a disorganised amalgamation of retinal, glial, vascular and RPE cells. Variations in the relative proportions of these components contribute to the heterogeneous clinical appearance.

Although clinical appearance is often diagnostic, ultrasonography, OCT and FFA are useful modalities for demonstrating the cardinal features of this condition thereby helping one to confirm the diagnosis. ICG angiography is generally normal in CHR-RPE except for mild hyperfluorescence corresponding to the tumour location in the late phase. ${ }^{5}$ It is however a valuable tool for excluding other choroidal pathologies. Long-term follow-up of CHR-RPE should be carried out with the aid of photography and OCT to monitor the dimensions of the tumour and evaluate the extent of vitreoretinal fibrosis or subretinal fluid. Tractional distortion of the fovea or subretinal fluid secondary to vitreomacular traction can lead to secondary visual loss. Although there is no established consensus on the management of these complications, pars plana vitrectomy with release of traction, with or without membrane peeling, has been performed in such cases with a $60 \%$ rate of visual improvement. ${ }^{6}$ Intravitreal triamcinolone and laser photocoagulation of the vascular component may also have a role in the management of CHR-RPE. ${ }^{8}$

In a review of 53 patients with CHR-RPE reported from 1952 to 1988, six showed apparent increase in the size of the lesion, five of whom underwent enucleation for presumed melanomas but were found to be combined hamartomas histologically. ${ }^{2}$ The most significant predictor of poor vision in CHR-RPE is involvement of the disc and macula. Documented locations of CHR-RPE are $76 \%$ juxtapapillary, $17 \%$ macular and $7 \%$ peripheral. Secondary visual loss can occur in $24 \%$ of cases due to tractional distortion of the fovea, subretinal fluid or pre-retinal neovascularisation. ${ }^{9} 10$

The majority of CHR-RPE appears to be sporadic, however it has been reported to occur in association with type II (and to a lesser extent type I) neurofibromatosis, nasopharyngeal angiofibroma, Gorlin and Poland syndromes. ${ }^{11-15}$ General investigations should be guided by clinical suspicion based on systemic review.

\section{Learning points}

- Combined hamatoma of the retina and retinal pigment epithelium (CHR-RPE) is a rare and benign ocular tumour.

- CHR-RPE is generally sporadic but can occasionally occur as part of neurofibromatosis, nasopharyngeal angiofibroma, Gorlin or Poland syndrome.

- Imaging modalities, in particular, B-scan ultrasonography, optical coherence tomography (OCT) and fundus fluorescein angiography (FFA), should be used to distinguish CHR-RPE from malignant chorioretinal lesions, in particular choroidal melanoma and retinoblastoma.

- Long-term follow-up should be carried out with the aid of photography and OCT to monitor the dimensions of the tumour and evaluate the extent of vitreoretinal fibrosis or subretinal fluid.

Competing interests None.

Patient consent Obtained.

\section{REFERENCES}

1. Ting TD, McCuen BW II, Fekrat S. Combined hamartoma of the retina and retinal pigment epithelium: optical coherence tomography. Retina 2002;22:98-101.

2. Font RL, Moura RA, Sheltar DJ, et al. Combined hamartoma of the sensory retina and retinal pigment epithelium. Retina 1989;9:302-11.

3. Shields CL, Thangappan A, Hartzell K, et al. Combined hamartoma of the retina and retinal pigment epithelium in 77 consecutive patients visual outcome based on macular versus extramacular tumor location. Ophthalmology 2008:115:2246-52.

4. Gass JDM. An unusual hamartoma of the pigment epithelium and retina simulating choroidal melanoma and retinoblastoma. Trans Am Ophthalmol Soc 1973;71:171-83.

5. Moschos M, Ladas ID, Zafirakis PK, et al. Recurrent vitreous hemorrhages due to combined pigment epithelial and retinal hamartoma: natural course and indocyanine green angiographic findings. Ophthalmologica 2001;215:66-9. 


\section{BMJ Case Reports}

6. Cohn AD, Quiram PA, Drenser KA, et al. Surgical outcomes of epiretinal membranes associated with combined hamartoma of the retina and retinal pigment epithelium. Retina 2009;29:825-30

7. Zhang $\mathbf{X}$, Dong $F$, Dai $R$, et al. Surgical management of epiretinal membrane in combined hamartomas of the retina and retinal pigment epithelium. Retina 2010;30:305-9.

8. Nam DH, Shin KH, Lee DY, et al. Vitrectomy, laser photocoagulation, and intravitreal triamcinolone for combined hamartoma of the retina and retinal pigment epithelium. Ophthalmic Surg Lasers Imaging 2010;9:1-4.

9. Schachat AP, Shields JA, Fine SL, et al. Combined hamartomas of the retina and retinal pigment epithelium. Ophthalmology 1984;91:1609-15.

10. Helbig $\mathbf{H}$, Niederberger $\boldsymbol{H}$. Presumed combined hamartoma of the retina and retinal pigment epithelium with preretinal neovascularization. Am J Ophthalmol 2003;136:1157-9.
11. Sivalingam A, Augsburger J, Perilongo G, et al. Combined hamartoma of the retina and retinal pigment epithelium in a patient with neurofibromatosis type 2. J Pediatr Ophthalmol Strabismus 1991;28:320-2.

12. Tsai $\mathbf{P}, \mathbf{O}$ 'Brien JM. Combined hamartoma of the retina and retinal pigment epithelium as the presenting sign of neurofibromatosis-1. Ophthalmic Surg Lasers 2000;31:145-7.

13. Fonseca RA, Dantas MA, Kaga T, et al. Combined hamartoma of the retina and retinal pigment epithelium associated with juvenile nasopharyngeal angiofibroma. Am J Ophthalmol 2001;132:131-2.

14. De Potter $\mathbf{P}$ Stanescu D, Caspers-Velu L, et al. Photo essay: combined hamartoma of the retina and retinal pigment epithelium in Gorlin syndrome. Arch Ophthalmol 2000;118:1004-5.

15. Stupp T, Pavlidis M, Bochner T, et al. Poland anomaly associated with ipsilateral combined hamartoma of retina and retinal pigment epithelium. Eye (London) 2004:18:550-2.

Copyright 2012 BMJ Publishing Group. All rights reserved. For permission to reuse any of this content visit http://group.bmj.com/group/rights-licensing/permissions.

BMJ Case Report Fellows may re-use this article for personal use and teaching without any further permission.

Please cite this article as follows (you will need to access the article online to obtain the date of publication).

Xue K, Mellington F, Gout I, Rokerya S, Olurin OI, El-Amir A. Combined hamartoma of the retina and retinal pigment epithelium. BMJ Case Reports 2012;10.1136/bcr-2012-006944, Published XXX

Become a Fellow of BMJ Case Reports today and you can:

- Submit as many cases as you like

- Enjoy fast sympathetic peer review and rapid publication of accepted articles

- Access all the published articles

- Re-use any of the published material for personal use and teaching without further permission

For information on Institutional Fellowships contact consortiasales@bmjgroup.com

Visit casereports.bmj.com for more articles like this and to become a Fellow 